\title{
Canadian high-energy neutron spectrometry system (CHENSS)
}

\author{
M.B. Smith*† \\ Bubble Technology Industries \\ P.O. Box 100, Chalk River, Ontario, Canada KOJ $1 \mathrm{JO}$ \\ E-mail: smithm@bubbletech.ca
}

\author{
H.R. Andrews, E.T.H. Clifford, H. Ing, V.T. Koslowsky, R.A. Noulty, M. Zhang \\ Bubble Technology Industries \\ P.O. Box 100, Chalk River, Ontario, Canada KOJ $1 \mathrm{JO}$
}

\section{L.G.I. Bennett, M.L. Boudreau, A.R. Green, B.J. Lewis}

Royal Military College of Canada

P.O. Box 17000, Kingston, Ontario, Canada K7K 7B4

\section{R. Nolte, S. Röttger}

Physikalisch-Technische Bundesanstalt

Braunschweig 38116, Germany

\begin{abstract}
The Canadian high-energy neutron spectrometry system (CHENSS) has been constructed in order to accurately characterize the fluence and energy distribution of high-energy neutrons encountered on space missions in low-Earth orbit. The CHENSS is a proton-recoil spectrometer based on a cylindrical gelled scintillator, with pulse-shape discrimination properties comparable to those of a liquid scintillator, completely surrounded by thin plastic panels which can be used to veto coincident events due to charged particles. The CHENSS has been irradiated by monoenergetic neutron beams, with energies up to $19 \mathrm{MeV}$, at the Physikalisch-Technische Bundesanstalt. Comparison of the data with fluence determinations performed in parallel to the CHENSS measurements shows good consistency and demonstrates the efficacy of the spectrometer for measurements in space.
\end{abstract}

International Workshop on Fast Neutron Detectors and Applications

April, 3 - 6, 2006

University of Cape Town, South Africa

\footnotetext{
*Speaker.

${ }^{\dagger}$ Project funded by the Canadian Space Agency (Life and Physical Sciences Directorate).
} 


\section{Introduction}

Radiation protection associated with human spaceflight is an important issue which becomes even more vital as both the length of the mission and the distance from Earth increase. Given the recent rejuvenation of interest in long-duration space travel, for example on the International Space Station (ISS) and planned moon and Mars missions, understanding of radiation issues is paramount. Radiation in space is a mixed field, generally divided into two categories: galactic cosmic rays (GCR) produced in supernova explosions outside the solar system and solar particle events (SPE) from the sun. In low-Earth orbit (LEO), protons and electrons trapped in the Van Allen radiation belts are a major component of the radiation field, especially when travelling above an area off the coast of Brazil referred to as the South Atlantic anomaly (SAA).

Neutrons emitted by the sun do not travel great distances in space because the neutron decays with a half-life of approximately ten minutes. Neutrons encountered in LEO are instead produced by nuclear reactions when GCR or trapped protons collide with or stop in the walls of the spacecraft or by neutron albedo after GCR are incident on the Earth's atmosphere. Neutron dose measurements on Russian space flights and on the space shuttle, performed with a bubble detector spectrometer, have indicated a large high-energy neutron flux above $10 \mathrm{MeV}[1,2]$. Uncertainty in the bubble detector response, however, prohibited the acquisition of reliable spectral information beyond that point. It has been shown experimentally that these high-energy neutrons contribute a significant fraction, perhaps as much as $60 \%$, of the total dose equivalent received by astronauts [3]. These observations are in agreement with theoretical calculations [4] which suggest that half of the neutron flux in LEO is above $10 \mathrm{MeV}$ in energy.

In support of the effort to reliably measure and predict radiation dose to astronauts, Bubble Technology Industries Inc. (BTI), with the support of the Canadian Space Agency (CSA), has developed an experiment aimed at measuring the neutron flux and energy spectrum in LEO from a few $\mathrm{MeV}$ up to $100 \mathrm{MeV}$. This information is also essential to optimize radiation shielding scenarios and for development of countermeasures. Furthermore, high-energy neutron spectra are now used as input to compute radiation effects (e.g. single-event upsets) on electronic equipment used in spacecraft. The Canadian high-energy neutron spectrometry system (CHENSS) is a proton-recoil spectrometer based on a cylindrical gelled scintillator surrounded by plastic detectors which can be used to veto coincident events due to charged particles. In this paper we describe the detector design and its performance characteristics, as well as the results of irradiation experiments performed using beams of monoenergetic neutrons at the Physikalisch-Technische Bundesanstalt (PTB). The detector concepts and technical details of the CHENSS have previously been described in some detail in Ref. [5].

\section{Spectrometer design}

Investigation of high-energy neutrons in space necessarily requires a new type of detection material because fast neutron detectors are traditionally made of liquid compounds that are considered to be hazardous cargo for space flights. To this end, BTI has developed a visco-elastic polymer, which will not spill or run if its container is opened, for use in the CHENSS. The spectrometer has been constructed to be flown autonomously on-board the space shuttle as part of NASA's Get-Away 
Special (GAS) programme. The GAS format imposes severe constraints on the spectrometer design and operation, limiting its size and imposing power, temperature, vibration and safety constraints.

Neutrons entering the active volume of the spectrometer interact in various ways with hydrogen and carbon in the organic elastomer. For incident neutron energies below $20 \mathrm{MeV}$, the dominant reaction is elastic neutron-proton scattering, which has a high cross-section for which the energy dependence is accurately known. Above $20 \mathrm{MeV}$, neutron-carbon reactions compete with neutronproton scattering, which makes the response function considerably more complicated. Scattered protons produce scintillation light with a very different time signature from that of $\gamma$ radiation. Pulse-shape analysis of the light output from the gelled scintillator can thus be used to discriminate between events due to neutrons and those due to background $\gamma$ rays. The BTI-developed material has pulse-shape discrimination properties similar to those of the widely-used NE-213 and BC-501A liquid scintillators.

The gelled scintillator is housed in a cylindrical stainless steel pressure vessel, $12.7 \mathrm{~cm}$ high and $12.7 \mathrm{~cm}$ in diameter, providing enough material to reliably detect $100 \mathrm{MeV}$ neutrons. The active detection volume has been designed to have the same geometry as a Japanese BC-501A liquid scintillator [6] with a known response function for neutrons with energies up to $120 \mathrm{MeV}$, allowing a comparison to the response function of the CHENSS. The pressure vessel can be heated, if needed, to maintain its temperature above $-5{ }^{\circ} \mathrm{C}$ (the elastomer becomes opaque below $-10{ }^{\circ} \mathrm{C}$ ). Four photomultiplier tubes (PMT's) are used to collect the light output from the gelled scintillator as shown in Fig. 1(a). This assembly is covered by a large mu-metal shield to protect the PMT bases from gain shifts due to the potential presence of magnetic fields.
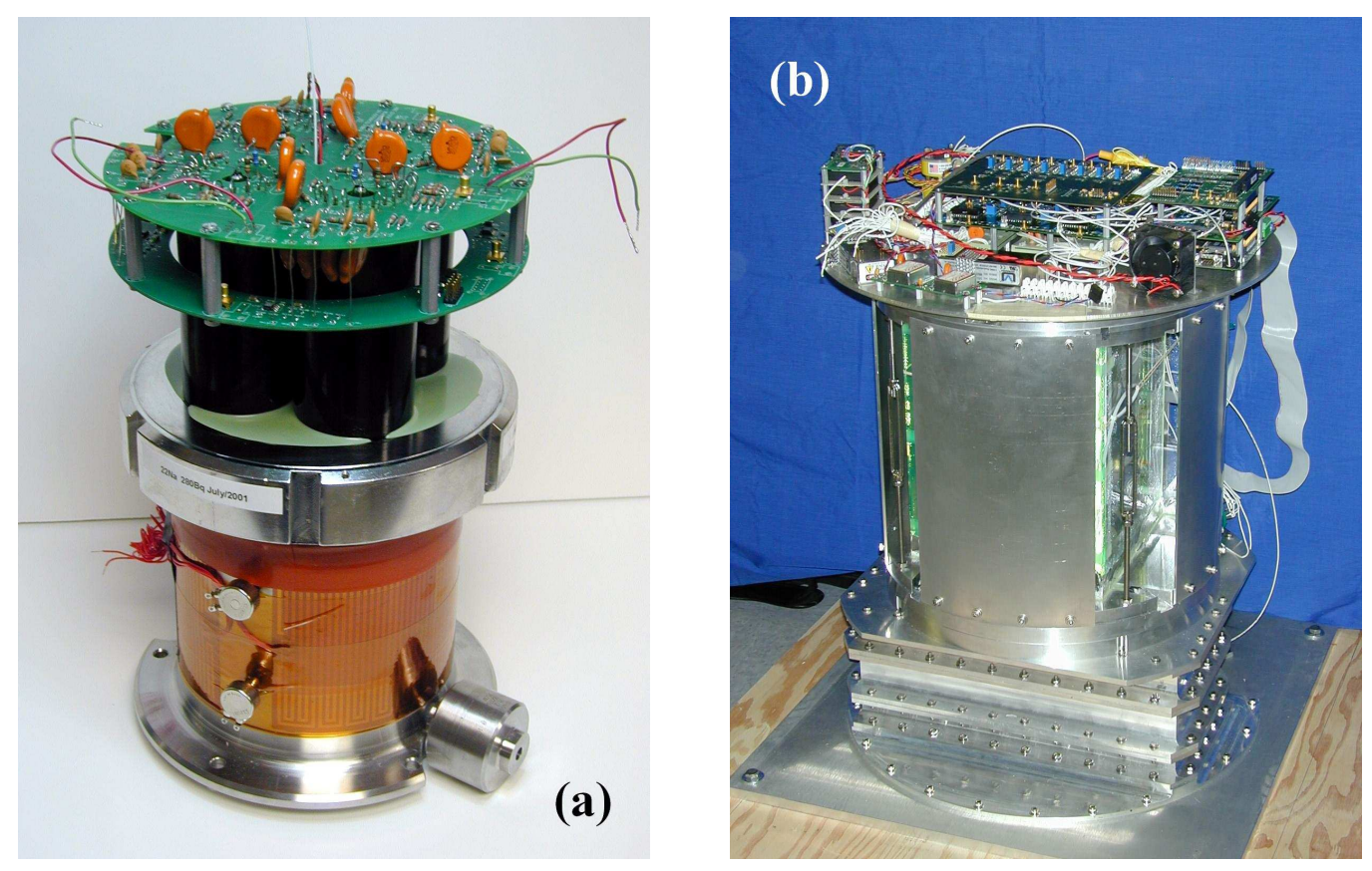

Figure 1: Photographs of (a) the housing for the visco-elastic scintillator and its photomultiplier tubes and (b) the completed spectrometer. 
The background from GCR and trapped protons cannot be eliminated by pulse-shape analysis since scintillation pulses due to these events exhibit the same signature as pulses due to neutroninduced recoil protons. Above $100 \mathrm{MeV}$, this background must be rejected efficiently because the GCR and trapped protons can be ten times more plentiful than neutrons of the same energy. In order to reject these events, the elastomer is completely surrounded by a rectangular box of eight plastic scintillators, each $1 \mathrm{~cm}$ thick with a wavelength-shifting bar on one of its sides. There are openings where necessary for cable feed-throughs, and so the top and bottom of the box arrangement are each equipped with two panels to provide full solid-angle coverage. Protons from the background interact in both the plastic and gelled scintillators and can thus be discriminated from neutroninduced events with veto efficiency close to $100 \%$. A photograph of the completed CHENSS is displayed in Fig. 1(b). The scintillation detectors and photomultipliers are housed in the centre of the spectrometer, between the data acquisition electronics (upper level) and the battery box used to power the spectrometer. The total power consumption of 50 watts is provided by 96 alkaline $\mathrm{D}$ batteries.

Detection of neutrons in the required range of a few $\mathrm{MeV}$ to $100 \mathrm{MeV}$ requires a factor of 300 in light output dynamic range, largely because of the non-linearity of light output from recoil protons. In order to achieve this, each event is recorded at three different gain settings (referred to as low-, mid- and high-gain). The summed signal from the PMT anodes is used to define a trigger and the summed signal from each of the last three PMT dynodes is recorded for analysis of the event. The gain increases by a factor of approximately seven at each dynode stage, thus providing an overall increase in output dynamic range of more than 300. For each of the three outputs, the signal amplitude and a shape signal are recorded (on two hard drives) so that pulse-shape analysis can be performed. The amplitude is integrated over two ranges ( 0 - $30 \mathrm{~ns}$ and $0-300 \mathrm{~ns})$ and the shape signal, a measure of the long component of light scintillation, is proportional to the difference of the two time integrals.

The severe temperature variations encountered in space dictate that monitoring of the PMT gains and environmental conditions be performed periodically. An internal energy calibration is provided by a $200-\mathrm{Bq}{ }^{22} \mathrm{Na}$ source, mounted to the tip of a thermocouple in the centre of the scintillating vessel. Furthermore, two green light-emitting diodes (LED's) are pulsed regularly to provide signals for relative gain calibration of the three dynode signals. The hit pattern of the veto panels is also recorded so that cosmic-ray events can be rejected, along with scaler values that monitor counting rates (allowing for accurate dead-time corrections), system voltages, temperatures and time. The data recorded ensures that the neutron information can be correlated with the spacecraft position in space and time, and the level of diagnostic information reduces the risk of the data being uninterpretable in post-flight analysis.

\section{Irradiation with monoenergetic neutron beams}

The monoenergetic neutron beams produced at the PTB accelerator facility have been wellcharacterized [7] and are routinely available as standard reference fields for calibration purposes. The CHENSS was irradiated by neutron beams with four incident energies, produced at $0^{\circ}$ emission angle in light-ion reactions as defined in the ISO 8592 standard, in a low-scatter experimental hall. The ${ }^{3} \mathrm{H}(\mathrm{p}, \mathrm{n})^{3} \mathrm{He}$ reaction was used to produce $2.5-\mathrm{MeV}$ neutrons, $5-\mathrm{MeV}$ beams were produced by 
the ${ }^{2} \mathrm{H}(\mathrm{d}, \mathrm{n})^{3} \mathrm{He}$ reaction and the ${ }^{3} \mathrm{H}(\mathrm{d}, \mathrm{n}){ }^{4} \mathrm{He}$ reaction was used, with different deuteron energies, to produce both 14.8- and 19-MeV neutrons. Data were collected at each beam energy both with and without a shadow cone between the neutron production target and the CHENSS, in order to correct for scattering of neutrons within the room. For the 5- and 19-MeV neutron fields the deuterons used in the reaction have sufficiently high energy that it is possible for them to produce neutrons in reactions not associated with the target. To correct for these reaction products further data were taken without the deuterium and tritium targets in place, again with and without the shadow cone. The data were analyzed using custom-built analysis routines written using the MATLAB [8] software package.

Two-dimensional energy-shape spectra recorded at each beam energy are presented in Fig. 2. Background components, due to scattered neutrons and neutrons produced without a target, have been subtracted after normalizing in time (including a dead-time correction due to different counting rates). Figure 2 demonstrates the separation of neutrons and $\gamma$ rays by their different pulse shapes, neutron events having a longer shape signal than $\gamma$-ray events, as indicated in panel (a). The figure also illustrates the utility of recording each event with three dynamic ranges, with panels (a) and (b) showing spectra recorded at the high-gain setting and panels (c) and (d) showing mid-gain spectra. A polygonal coincidence window has been applied to the energy-shape spectra to isolate neutron events from the $\gamma$-ray background. The resulting neutron spectrum has been
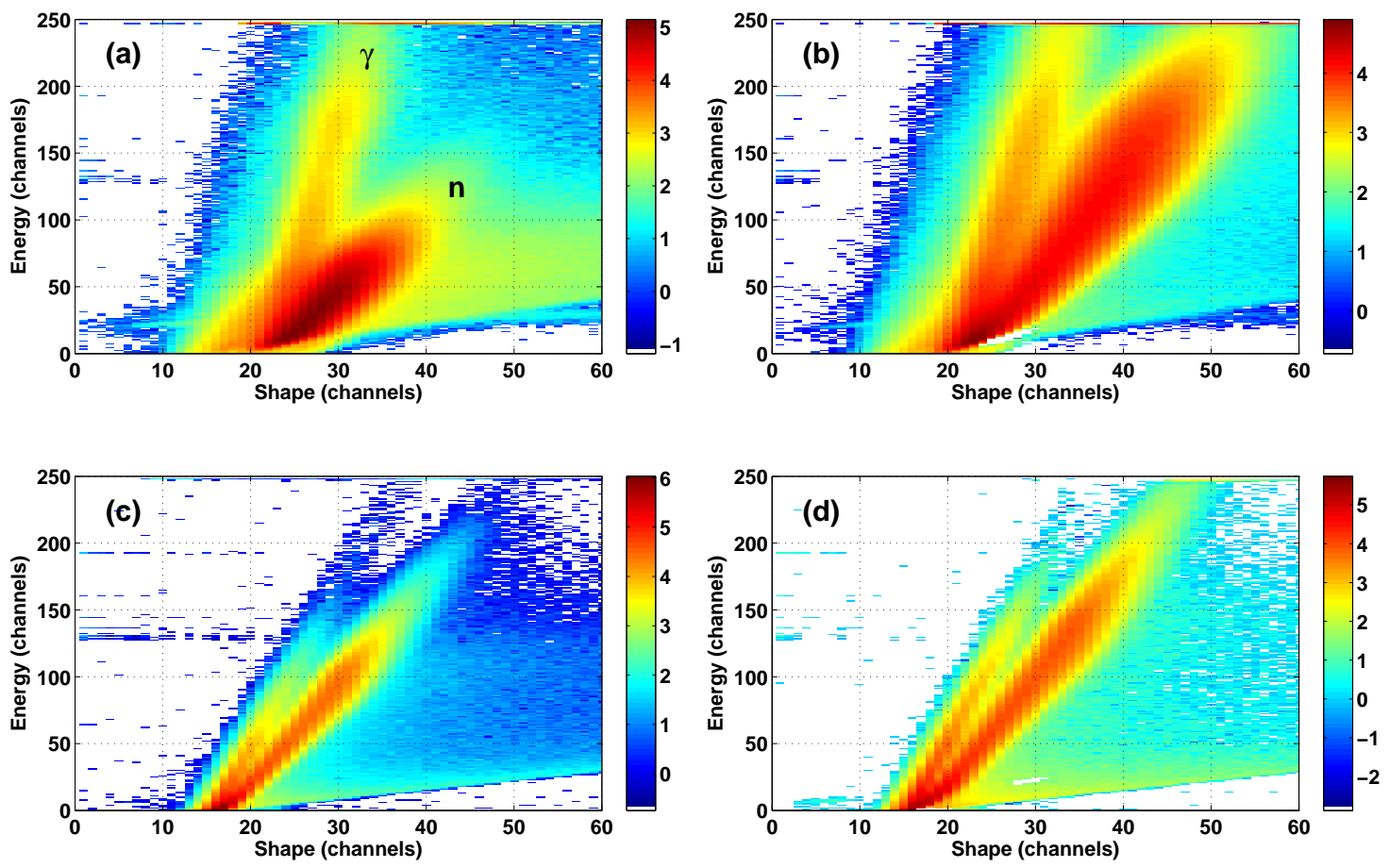

Figure 2: Total energy-shape spectrum measured by the CHENSS, after subtraction of background components, following irradiation with (a) $2.5-\mathrm{MeV}$, (b) $5-\mathrm{MeV}$, (c) $14.8-\mathrm{MeV}$ and (d) $19-\mathrm{MeV}$ neutron beams. It should be noted that panels (a) and (b) show high-gain spectra whereas panels (c) and (d) show mid-gain spectra. The intensity (colour) scale is in logarithmic units. 
unfolded to provide a measurement of the total neutron fluence incident on the spectrometer.

The measured spectra were unfolded using the response matrix determined for the cylindrical liquid scintillator discussed in Ref. [6], which has the same size as the gelled scintillator used in the CHENSS. The generalized inverse of the response matrix was determined using the singular value decomposition technique. Examples of unfolded neutron spectra are displayed in Fig. 3 for the 14.8- and 19-MeV neutron fields. The peak area in these spectra is related to the neutron fluence incident on the detector and can be compared to fluence determinations performed in parallel using stable long counters $\left(\mathrm{BF}_{3}\right.$ proportional tubes) provided by $\mathrm{PTB}$. The two independent fluence measurements are summarized in Tab. 1. The peak area in the unfolded CHENSS spectrum was converted to fluence, $\Phi_{\text {CHENSS }}$, by dividing by the active area of the gelled scintillator (assumed to be a $12.7 \times 12.7 \mathrm{~cm}$ square). The long-counter fluence, $\Phi_{\text {РTВ }}$, results from subtracting background measurements due to scattering and non-target reactions, and normalizing to the dead-time corrected measurement time of the CHENSS. The fluence determinations are in excellent agreement for the 14.8- and 19-MeV fields but the agreement lessens at the lower neutron energies, with the CHENSS fluences lower than the long-counter measurements for both the 2.5- and 5-MeV neutron fields. This is understood since the neutron- $\gamma$ discrimination is difficult at lower energies and the coincidence window may exclude some neutrons. The unfolding process is also unreliable in this regime because the Nakao response matrix is primarily intended for use with high-energy neutrons and contains insufficient information at low energies.

\section{Conclusion}

The CHENSS has been constructed with the aim of extending neutron radiation measurements in space into the high-energy $(>10 \mathrm{MeV})$ regime currently inaccessible to instruments such as the bubble detector. The spectrometer is currently in a testing phase both for performance and space qualification. As part of this testing the device has been irradiated with monoenergetic neutron beams, with energies ranging from 2.5 to $19 \mathrm{MeV}$, at the PTB accelerator facility. Good agreement is obtained between fluence measurements performed with the CHENSS and parallel determinations using PTB's long counters. Similar tests with higher-energy neutron fields are desirable and
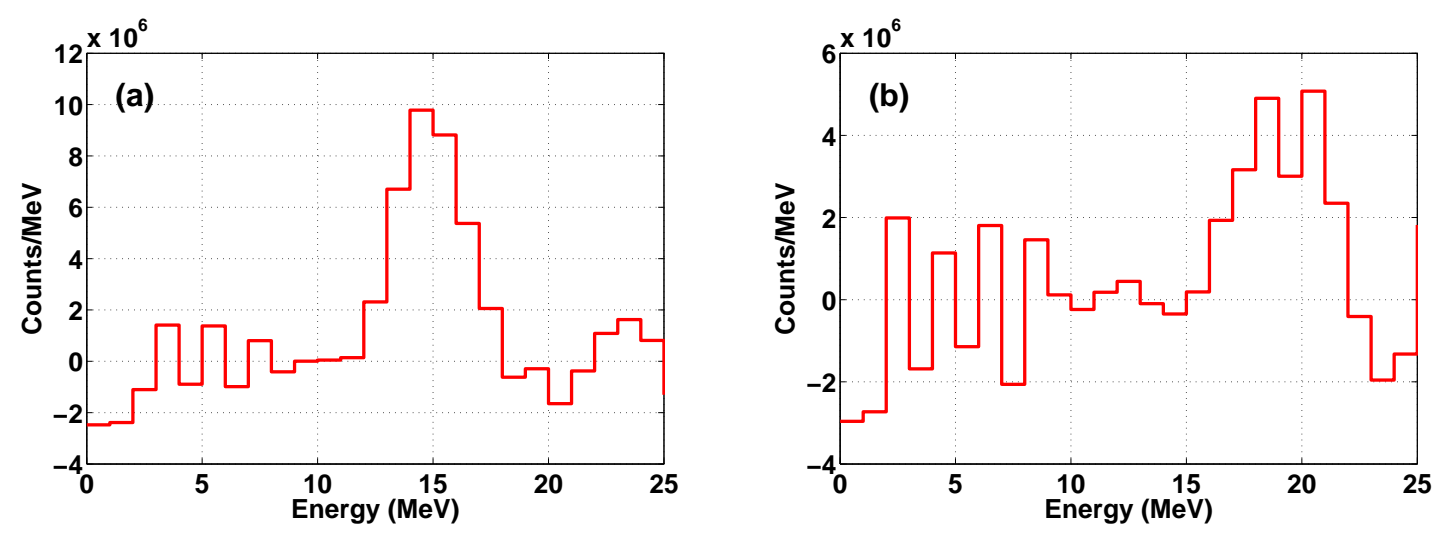

Figure 3: Unfolded neutron spectra for (a) 14.8-MeV and (b) 19-MeV fields. 


\begin{tabular}{ccc}
\hline \hline Neutron energy $(\mathrm{MeV})$ & $\Phi_{\text {PTB }}\left(10^{5}\right.$ neutrons $\left./ \mathrm{cm}^{2}\right)$ & $\Phi_{\text {CHENSS }}\left(10^{5}\right.$ neutrons $\left./ \mathrm{cm}^{2}\right)$ \\
\hline \hline 2.5 & $3.3(3)$ & $2.0(4)$ \\
5.0 & $2.2(2)$ & $1.9(4)$ \\
14.8 & $2.2(3)$ & $2.2(2)$ \\
19.0 & $1.4(2)$ & $1.3(2)$ \\
\hline \hline
\end{tabular}

Table 1: Comparison of fluence $(\Phi)$ measurements performed using the PTB long counters and the CHENSS. Uncertainties are given in parentheses.

it is planned that the CHENSS will be irradiated with $30-200-\mathrm{MeV}$ neutron beams at the Institut de Physique Nucléaire of the Université catholique de Louvain (Belgium) and the iThemba Laboratory for Accelerator-Based Sciences (South Africa).

\section{References}

[1] H. Ing, Radiat. Meas. 33, 275 (2001).

[2] A.R. Green, H.R. Andrews, L.G.I. Bennett, E.T.H. Clifford, H. Ing, G. Jonkmans, B.J. Lewis, R.A. Noulty, E.A. Ough, Acta Astronautica 56, 949 (2005).

[3] G.D. Badhwar (Ed.), Recommendations of the Predictions and Measurements of Secondary Neutrons in Space Workshop, NASA/Johnson Space Center, September 28-30, 1998; published in part in Radiat. Meas. 33 (2001).

[4] T.W. Armstrong and B.L. Colborn, Radiat. Meas. 33, 229 (2001).

[5] G. Jonkmans et. al., Acta Astronautica 56, 975 (2005).

[6] N. Nakao, T. Nakamura, M. Baba, Y. Uwamino, N. Nakanishi, H. Nakashima, S. Tanaka, Nucl. Instrum. Meth. Phys. Res. Sect. A 362, 454 (1995).

[7] R. Nolte et. al., Radiat. Protect. Dosim. 110, 97 (2004).

[8] http://www.mathworks.com 